\title{
Respiratory complex I: hop (structure), step (reaction mechanism) and jump (potential remedy)
}

\author{
Takao Yagi • Akemi Matsuno-Yagi
}

Published online: 2 July 2014

(C) Springer Science+Business Media New York 2014

This is the third mini-review series on respiratory complex I published in the Journal of Bioenergetics and Biomembranes. The first series was released in 1993 under the title, "The Most Complex Complex" (ed. Ohnishi). The gene cluster encoding bacterial complex I had been cloned and sequenced. The second one came out in 2001 with the title, "An L-shaped Black Box" (ed. Yagi). At that time, 3D structures of the other respiratory complexes had been solved, leaving complex I as the only one whose structure was in a black box (see Fig. 1). We have since witnessed an overwhelming advancement in the research of complex I. Publishing another review series was long overdue especially because the latest progress marked one of the most exciting times in the long history of complex I research. Above all, we saw the structure of complex I at the atomic level for the first time which revealed an astonishing machinery of this huge enzyme complex.

One of the advantages of putting together multiple papers into a mini-review series is that we can see innovative strategies and tactics presented by the authors with different disciplinary backgrounds and viewpoints. Interest in complex I could be purely scientific or more clinically oriented. We hope we have covered the most important areas which include structure, reaction mechanism, and potential remedy. These areas are, of course, not independent but rather inter-dependent.

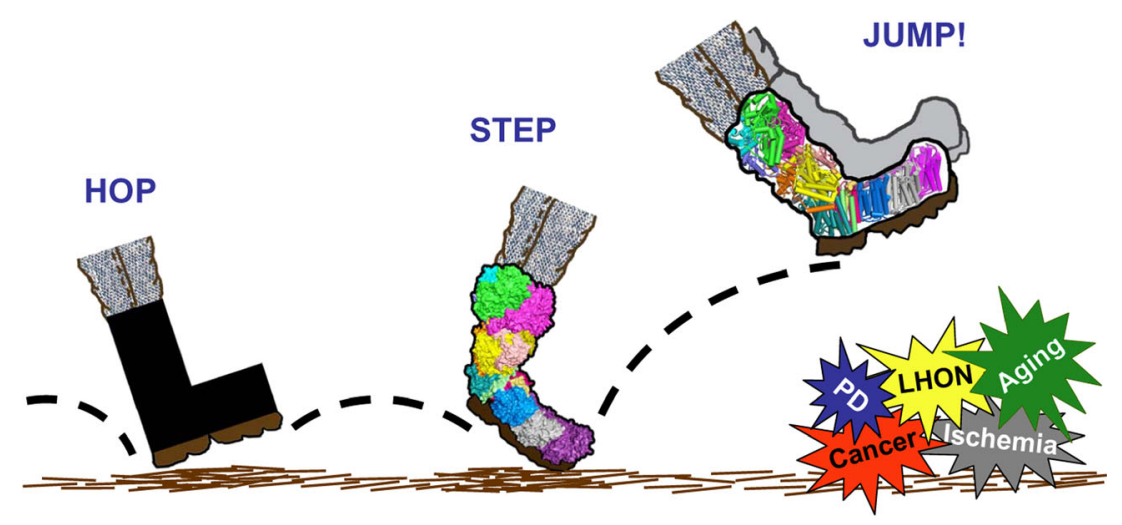

Fig. 1 The start of the complex I research goes back to the ' 60 s. It had a long approach run. This was an important phase that developed momentum for the next phases. There were many valuable biochemical and biophysical discoveries. Then came a take-off. The hop phase was a great forward

T. Yagi $(\bowtie) \cdot$ A. Matsuno-Yagi

The Scripps Research Institute, 10550 N. Torrey Pines Rd., La Jolla, CA 92037, USA

e-mail: yagi@scripps.edu

A. Matsuno-Yagi

e-mail: ayagi@scripps.edu motion with elucidation of the three-dimensional structure. As the take-off leg lands in the step phase, a number of proposals for the mechanism started bursting. The energy accumulated now must explode into the jump phase to tackle the challenging tasks of solving human diseases

We have organized this mini-series with 10 distinct author groups as follows:

On the basis of the known 3D structure combined with bioengineering techniques, the coupling mechanisms of complex I was discussed by Sazanov, Friedrich, Nakamaru-Ogiso et al., and Sato et al. Furthermore, the complex I family, 
sodium-pumping bacterial NADH:quinone oxidoreductase was covered by Barquera.

Complex I is located in a wide range of organisms such as bacteria, plant and mammalian mitochondria. It is known that Trypanosomatids contain complex I with multifunctions. Duarte and Tomas summarized its current knowledge.

Complex I is a unique enzyme in that chemical modifications and assembly factors could regulate the structure and function. Murai and Miyoshi, Vartak et al. discussed this area.

Dysfunction of complex I is well known to cause certain mitochondrial diseases. Today, complex I is linked to more common events such as aging and ischemia. Hur et al. and Pepe et al. covered these subjects.

We thank all active investigators who contributed to this mini-review series. 